\title{
Influence of Various Seed Amelioration Techniques on Physio-Biochemical Changes during Seed Deterioration in Aged Seeds of Soybean [Glycine max (L.) Merill] Mini Core Set
}

\author{
K. Shruthi", R. Siddaraju, P.J. Devaraju, N. Nethra, \\ Jayarame Gowda and Y.A. Nanja Reddy \\ Department of Seed Science and Technology, College of Agriculture, GKVK, UAS, \\ Bengaluru-560 065, Karnataka, India \\ *Corresponding author
}

\section{A B S T R A C T}

\section{Keywords \\ Seed deterioration, Seed germination, Membrane injury index, Seedling vigour index, Electrical conductivity \\ Article Info \\ Accepted: \\ 04 August 2018 \\ Available Online: \\ 10 September 2018}

The laboratory study was conducted to ameliorate the aged seeds of soybean mini core set by using various antioxidants and osmotics viz., $\alpha$-tocopherol (1\%), ascorbic acid (1\%), potassium iodide $(2.5 \%)$ and PEG-6000 (1\%). Results revealed that the amelioration with $\alpha$-tocopherol $(1 \%)$ and ascorbic acid $(1 \%)$ treatments showed a marked increase in the seed physio-biochemical parameters like seed germination (from $61 \%$ to $74 \%$ ), seedling vigour index I (from 783 to 1292), seedling vigour index II (from 330 to 446) and decrease in electrical conductivity $(1.80 \mathrm{dS} / \mathrm{cm}$ to $1.63 \mathrm{dS} / \mathrm{cm})$ and membrane injury index (53.9 \% to $51.8 \%$ ) compared to untreated aged seeds. Results of these amelioration treatments indicates the repair and re-synthesizing ability of antioxidants and their role in stabilizing seed deterioration damages in aged seeds. The data's were statistically analyzed by analysis of variance $(\mathrm{P} \leq 0.01)$.

\section{Introduction}

Soybean [Glycine $\max$ (L.) Merrill.] is an important pulse crop popularly known as miracle crop due to its multiple uses that has around 40 per cent protein and 20 per cent oil in it.

The soybean (Glycine $\max$ L. Merril) was originated from eastern Asia/China, it is a member of Leguminacae family and cultivated soybean (Glycine max L. Merril) was derived from a wild progenitor Glycine ussuriensi. Sub species of soybean are Glycine gracilis and Glycine soja, cultivated soybean has genome size of 1.1 to $1.15 \mathrm{~Gb}$ with chromosome pair of twenty $(2 \mathrm{n}=40)$.

Soybean seed has been identified as poor storer, because of its delicate (thin) seed coat and vulnerable position of its embryo. Hence it is very much prone for seed deterioration losses. Though, seed deterioration is irreversible and inexorable but its rate and extent can be slowed down to some extent through seed amelioration techniques such as seed priming with antioxidants osmotics and salts etc. Beneficial effect of such seed 
treatment was reflected in better germination and early seedling growth accompanied by greater cellular membrane integrity, counteraction of lipid peroxidation and free radical chain reaction often found to be directly correlated with the maintenance of vigour and viability of seeds (Kapoor et al., 2011).

Therefore an attempt was made to explore such amelioration treatments to enhance performance of low vigour genotypes of soybean germplasm accessions. In the backdrop of this information the present study has been undertaken to study the effect of various amelioration techniques on aged seeds during seed deterioration.

\section{Materials and Methods}

The present study was conducted using artificially aged seeds of soybean mini core set (Table 1) which are having different vigour levels. This aged seeds were subjected to various amelioration treatments viz., $\mathrm{T}_{1}$ : Control; $\mathrm{T}_{2}$ : $\alpha$-tocopherol @ $1 \%$ (organic infusion); $\mathrm{T}_{3}$ : Ascorbic acid @ $1 \%$ soaking; $\mathrm{T}_{4}$ : Potassium iodide @ $2.5 \%$ soaking; $\mathrm{T}_{5}$ : PEG-6000@1\%.

As soybean seeds are prone to soaking injury, seed amelioration were done by placing the seeds between moist papers which were soaked in each treatment, instead of soaking directly in aqueous solution. Then, these seeds were thoroughly washed, surface dried under room temperature and used for further experiments.

\section{Accelerated ageing}

Fresh seeds (untreated) were subjected to artificial ageing (Anon., 2010) for a period of 10 days at $45{ }^{\circ} \mathrm{C}$ temperature and $95 \% \mathrm{RH}$. Samples were collected at 2 days interval for seed quality studies.

\section{Seed germination $(\%)$}

The laboratory germination test was conducted as per the ISTA rules (2010) using between paper method. Fifty seeds in eight replications were allowed to germinate at temperature of $25^{\circ} \mathrm{C}$ up to 8 days. The germination counts were recorded on $5^{\text {th }}$ and $8^{\text {th }}$ day and per cent germination was expressed on normal seedling basis.

\section{Seedling vigour index-I and II}

The vigor index I was determined by multiplying the percentage germination and total seedling length and whereas vigour index-II was determined by multiplying percentage germination with total seedling dry weight (Abdul Baki and Anderson, 1973)

Seedling vigour index $\mathrm{I}=$ germination $(\%) \mathrm{x}$ mean seedling length $(\mathrm{cm})$

Seedling vigour index $\mathrm{II}=$ germination $(\%) \mathrm{x}$ mean seedling dry weight $(\mathrm{mg})$

\section{Electrical conductivity $\left(\mathrm{dScm}^{-1}\right)$}

Electrical conductivity was measured as per the ISTA rules (2010). Fifty seeds of 4 replication were weighed on an analytical balance and soaked in $75 \mathrm{ml}$ of distilled water for 24 hours at $25 \pm 1^{\circ} \mathrm{C}$. The EC at $25 \pm 1^{\circ} \mathrm{C}$ was measured using conductivity meter.

\section{Membrane injury index}

Membrane injury index was calculated by the formula given by Blum and Ebercon (1981).

$$
\mathrm{MII}=\left(\mathrm{C}_{1} / \mathrm{C}_{2}\right) * 100
$$

Where

$\mathrm{C}_{1=}$ Electric conductivity at $40{ }^{0} \mathrm{C}$ for $30 \mathrm{~min}$.

$\mathrm{C}_{2}=$ Electric conductivity at $100^{\circ} \mathrm{C}$ for $10 \mathrm{~min}$. 


\section{Statistical analysis}

The experimental data was statistically analyzed by adopting the analysis of variance technique appropriate to design as per the methods outlined by Sundararaj et al., (1972) in computer. Critical differences were calculated at 1 per cent level, where ' $F$ ' test was significant. Germination percentages (original values) were transformed into square root transformation. The transformed values were used for statistical analysis.

\section{Results and Discussion}

The results revealed the significant effects of seed amelioration treatments on aged seeds of soybean mini core set. Among the various seed treatments used, the $\alpha$-tocopherol found to increase the seed germination in PB-5 (89 $\%), \operatorname{EC} 57042(87 \%)$ and TR-5 (86 \%) followed by ascorbic acid (88 \%) and potassium iodide $(86 \%)$, whereas, least or negligible influence was observed in PEG treated seeds (Table 2).

However, highest per cent increase in seed germination was reported for $\alpha$-tocopherol treatment in AT-156 (13\%) and JS-20-42 (13 $\%)$ followed by ascorbic acid (12\%) and potassium iodide $(6 \%)$ in JS-20-42. Whereas,
PEG showed a least performance of $\leq 5$ per cent enhancement in seed germination in almost all the genotypes (DS-72-244, EC101549 and TAS-92-34) compared to other treatments (Fig. 1).

These differences in response by different genotypes may because of variation in their seed biochemical composition i.e. genotypes having higher level of endogenous antioxidants do not respond well for exogenous antioxidants application. The similar results were reported by Taylor et al., (1998) and Kaya et al., (2006).

The present result of enhanced seed germination in low vigour genotypes through antioxidant treatment was supported by Bailly et al., (1998) and Kaya et al., (2006) in sunflower, they reported that priming of aged seeds with antioxidants progressively restores the initial germinative ability and reduces the level of lipid peroxidation.

Significant differences were recorded among the genotypes (aged) and seed treatments for seedling vigour index-I \& II. Among various seed treatments $\alpha$-tocopherol reported the higher SVI-I \& II in EC-57042 (1888) (574) and PB-5 (1801) (568) compared to control (1544) (538) (Table 3).

Fig.1 Per cent increase in seed germination through amelioration treatments in aged seeds of soybean genotypes

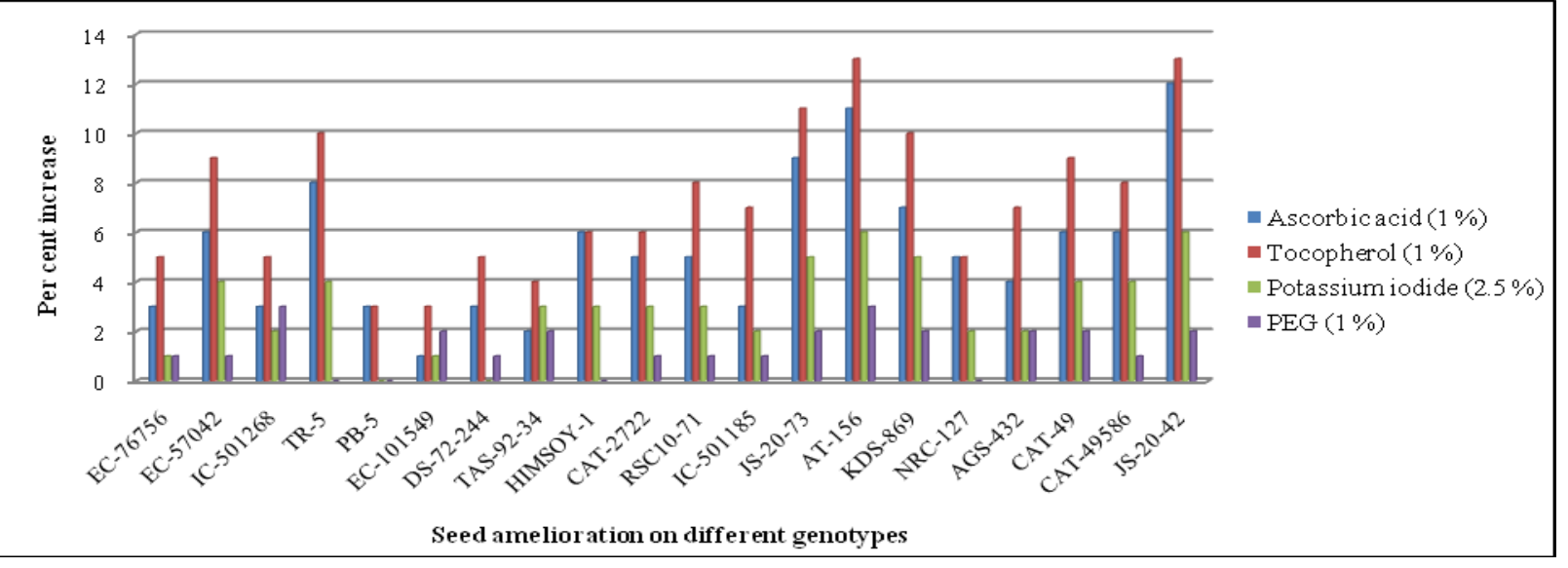


Table.1 List of soybean mini core set used for the study

\begin{tabular}{|c|l|l|l|}
\hline SI. No. & \multicolumn{1}{|c|}{ Genotypes } & SI. No. & \multicolumn{1}{|c|}{ Genotypes } \\
\hline $\mathbf{1}$ & EC-76756 & 11 & RSC10-71 \\
\hline $\mathbf{2}$ & EC-57042 & 12 & IC-501185 \\
\hline $\mathbf{3}$ & IC-501268 & 13 & JS-20-73 \\
\hline $\mathbf{4}$ & TR-5 & 14 & AT-156 \\
\hline $\mathbf{5}$ & PB-5 & 15 & KDS-869 \\
\hline $\mathbf{6}$ & EC-101549 & 16 & NRC-127 \\
\hline $\mathbf{7}$ & DS-72-244 & 17 & AGS-432 \\
\hline $\mathbf{8}$ & TAS-92-34 & 18 & CAT-49 \\
\hline $\mathbf{9}$ & HIMSOY-1 & 19 & CAT-49586 \\
\hline $\mathbf{1 0}$ & CAT-2722 & 20 & JS-20-42 \\
\hline
\end{tabular}

Table.2 Effect of seed amelioration on seed germination (\%) of aged seeds of Soybean mini core set

\begin{tabular}{|c|c|c|c|c|c|c|}
\hline \multirow[t]{2}{*}{$\begin{array}{l}\text { Sl. } \\
\text { No. }\end{array}$} & \multirow[t]{2}{*}{ Genotypes } & Control & $\begin{array}{c}\text { Ascorbic acid } \\
(1 \%)\end{array}$ & $\begin{array}{l}\text { Tocopherol } \\
(1 \%)\end{array}$ & $\begin{array}{l}\text { Potassium } \\
\text { iodide } \\
(2.5 \%)\end{array}$ & $\begin{array}{c}\text { PEG-6000 } \\
(1 \%)\end{array}$ \\
\hline & & 4 & 4 & 4 & 4 & 4 \\
\hline 1 & EC-76756 & $76(8.69)$ & $79(8.86)$ & $81(9.00)$ & 77(8.77) & $77(8.60)$ \\
\hline 2 & EC-57042 & $78(8.84)$ & $84(9.16)$ & $87(9.30)$ & $82(9.05)$ & $79(8.87)$ \\
\hline 3 & IC-501268 & $75(8.67)$ & $78(8.83)$ & $80(8.94)$ & 77(8.77) & $78(8.60)$ \\
\hline 4 & TR-5 & $76(8.73)$ & $84(9.16)$ & $86(9.25)$ & $80(8.92)$ & $76(8.74)$ \\
\hline 5 & PB-5 & $86(9.28)$ & $89(9.41)$ & $89(9.41)$ & $86(9.22)$ & $86(9.03)$ \\
\hline 6 & EC-101549 & $76(8.69)$ & 77(8.77) & $79(8.89)$ & $77(8.57)$ & $78(8.40)$ \\
\hline 7 & DS-72-244 & $75(8.67)$ & $78(8.80)$ & $80(8.94)$ & $75(8.54)$ & $76(8.37)$ \\
\hline 8 & TAS-92-34 & $75(8.44)$ & $77(8.75)$ & $79(8.86)$ & $78(8.57)$ & $77(8.40)$ \\
\hline 9 & HIMSOY-1 & $72(8.50)$ & $78(8.80)$ & $78(8.80)$ & 75(8.67) & $72(8.49)$ \\
\hline 10 & CAT-2722 & $71(8.62)$ & $76(8.69)$ & $77(8.77)$ & 74(8.43) & $72(8.26)$ \\
\hline 11 & RSC10-71 & $70(8.27)$ & $75(8.63)$ & $78(8.80)$ & $73(8.34)$ & $71(8.17)$ \\
\hline 12 & IC-501185 & $70(8.38)$ & $73(8.51)$ & $77(8.77)$ & $72(8.46)$ & $71(8.29)$ \\
\hline 13 & JS-20-73 & 64(8.33) & $73(8.51)$ & $75(8.63)$ & $69(8.27)$ & $66(8.11)$ \\
\hline 14 & AT-156 & $61(8.38)$ & $72(8.46)$ & $74(8.60)$ & $67(8.15)$ & 64(7.99) \\
\hline 15 & KDS-869 & 63(8.33) & $70(8.37)$ & $73(8.51)$ & $68(8.00)$ & $65(7.84)$ \\
\hline 16 & NRC-127 & $67(8.20)$ & $72(8.49)$ & $72(8.49)$ & $69(8.32)$ & $67(8.15)$ \\
\hline 17 & AGS-432 & $66(8.14)$ & $70(8.34)$ & $73(8.51)$ & 68(8.26) & $68(8.09)$ \\
\hline 18 & САT-49 & $65(8.08)$ & $71(8.28)$ & $74(8.60)$ & $69(8.28)$ & $67(7.94)$ \\
\hline 19 & CAT-49586 & $64(8.02)$ & $70(8.37)$ & 72(8.49) & $68(8.22)$ & $65(7.88)$ \\
\hline 20 & JS-20-42 & $60(7.77)$ & $72(8.46)$ & 73(8.51) & $66(8.13)$ & $62(7.87)$ \\
\hline \multirow{3}{*}{\multicolumn{2}{|c|}{$\begin{array}{c}\text { S. Em } \pm \\
\text { CD }(\mathbf{P}=0.01) \\
\text { CV }(\%)\end{array}$}} & 0.07 & 0.07 & 0.08 & 0.29 & 0.15 \\
\hline & & 0.26 & 0.29 & 0.34 & 0.73 & 0.34 \\
\hline & & 1.08 & 1.19 & 1.39 & 2.90 & 1.01 \\
\hline
\end{tabular}


Table.3 Effect of seed amelioration on seedling vigour index of aged seeds of soybean mini core set

\begin{tabular}{|c|c|c|c|c|c|c|c|c|c|c|c|}
\hline \multirow[t]{2}{*}{$\begin{array}{c}\text { SI. } \\
\text { No. }\end{array}$} & \multirow[t]{2}{*}{ Genotypes } & \multicolumn{2}{|c|}{ Control } & \multicolumn{2}{|c|}{$\begin{array}{c}\text { Ascorbic acid } \\
(1 \%)\end{array}$} & \multicolumn{2}{|c|}{$\begin{array}{c}\text { Tocopherol } \\
(1 \%)\end{array}$} & \multicolumn{2}{|c|}{$\begin{array}{c}\text { Potassium iodide } \\
(2.5 \%)\end{array}$} & \multicolumn{2}{|c|}{$\begin{array}{c}\text { PEG-6000 } \\
(1 \%)\end{array}$} \\
\hline & & SVI-I & SVI-II & SVI-I & SVI-II & SVI-I & SVI-II & SVI-I & SVI-II & SVI-I & SVI-II \\
\hline 1 & EC-76756 & 1346 & 447 & 1534 & 493 & 1638 & 522 & 1491 & 475 & 1418 & 470 \\
\hline 2 & EC-57042 & 1544 & 495 & 1771 & 545 & 1888 & 574 & 1632 & 525 & 1560 & 505 \\
\hline 3 & IC-501268 & 1352 & 462 & 1550 & 491 & 1645 & 512 & 1458 & 481 & 1459 & 471 \\
\hline 4 & TR-5 & 1457 & 477 & 1701 & 541 & 1792 & 566 & 1565 & 508 & 1519 & 478 \\
\hline 7 & DS-72-244 & 1319 & 464 & 1483 & 487 & 1585 & 514 & 1370 & 467 & 1370 & 470 \\
\hline 8 & TAS-92-34 & 1287 & 439 & 1551 & 479 & 1648 & 501 & 1533 & 484 & 1483 & 482 \\
\hline 9 & HIMSOY-1 & 1302 & 430 & 1537 & 470 & 1590 & 480 & 1430 & 451 & 1354 & 431 \\
\hline 10 & CAT-2722 & 1244 & 442 & 1467 & 462 & 1549 & 484 & 1416 & 447 & 1341 & 446 \\
\hline 11 & RSC10-71 & 1171 & 411 & 1373 & 463 & 1478 & 494 & 1267 & 446 & 1221 & 431 \\
\hline 15 & KDS-869 & 1042 & 380 & 1274 & 411 & 1366 & 445 & 1214 & 386 & 1107 & 391 \\
\hline 16 & NRC-127 & 976 & 365 & 1231 & 427 & 1274 & 451 & 1167 & 392 & 1099 & 369 \\
\hline 17 & AGS-432 & 1029 & 375 & 1258 & 418 & 1358 & 454 & 1193 & 396 & 1150 & 387 \\
\hline 18 & CAT-49 & 867 & 351 & 1204 & 405 & 1299 & 439 & 1153 & 382 & 1052 & 362 \\
\hline 19 & CAT-49586 & 836 & 351 & 1205 & 407 & 1283 & 436 & 1171 & 383 & 1045 & 357 \\
\hline 20 & JS-20-42 & 783 & 330 & 1231 & 420 & 1292 & 446 & 1140 & 374 & 1000 & 343 \\
\hline \multirow{3}{*}{\multicolumn{2}{|c|}{$\begin{array}{l}\text { S. Em } \pm \\
\text { CD }(P=0.01) \\
\text { CV }(\%)\end{array}$}} & 34.3 & 12.09 & 20.1 & 13.55 & 30.04 & 8.65 & 24.6 & 8.71 & 33.01 & 6.12 \\
\hline & & 97.7 & 34.41 & 81.2 & 54.56 & 120.8 & 34.81 & 99.3 & 35.05 & 132.8 & 24.6 \\
\hline & & 2.90 & 2.88 & 2.00 & 4.14 & 2.81 & 2.50 & 1.55 & 1.92 & 2.13 & 1.37 \\
\hline
\end{tabular}


Table.4 Effect of seed amelioration on seed coat membrane integrity on aged seeds of soybean mini core set

\begin{tabular}{|c|c|c|c|c|c|c|c|c|c|c|c|}
\hline \multirow[t]{2}{*}{$\begin{array}{l}\text { Sl. } \\
\text { No. }\end{array}$} & \multirow[t]{2}{*}{ Genotypes } & \multicolumn{2}{|c|}{ Control } & \multicolumn{2}{|c|}{$\begin{array}{c}\text { Ascorbic acid } \\
(1 \%)\end{array}$} & \multicolumn{2}{|c|}{$\begin{array}{c}\text { Tocopherol } \\
(1 \%)\end{array}$} & \multicolumn{2}{|c|}{$\begin{array}{c}\text { Potassium iodide } \\
(2.5 \%)\end{array}$} & \multicolumn{2}{|c|}{$\begin{array}{l}\text { PEG-6000 } \\
(1 \%)\end{array}$} \\
\hline & & $\begin{array}{c}\mathrm{EC} \\
(\mathrm{dS} / \mathrm{cm})\end{array}$ & $\begin{array}{l}\text { MII } \\
(\%)\end{array}$ & $\begin{array}{c}\mathrm{EC} \\
(\mathrm{dS} / \mathrm{cm})\end{array}$ & MII (\%) & $\begin{array}{c}\text { EC } \\
(\mathrm{dS} / \mathrm{cm})\end{array}$ & $\begin{array}{l}\text { MII } \\
(\%)\end{array}$ & $\begin{array}{c}\text { EC } \\
(\mathrm{dS} / \mathrm{cm})\end{array}$ & MII (\%) & $\begin{array}{c}\text { EC } \\
(\mathrm{dS} / \mathrm{cm})\end{array}$ & MII (\%) \\
\hline 2 & EC-57042 & 1.30 & 41.8 & 1.238 & 41.2 & 1.219 & 40.8 & 1.275 & 41.9 & 1.269 & 42.7 \\
\hline 3 & IC-501268 & 1.41 & 49.3 & 1.343 & 46.6 & 1.323 & 46.2 & 1.384 & 47.4 & 1.377 & 48.3 \\
\hline 6 & EC-101549 & 1.37 & 45.7 & 1.304 & 45.0 & 1.284 & 44.6 & 1.343 & 45.8 & 1.336 & 46.7 \\
\hline 7 & DS-72-244 & 1.43 & 45.1 & 1.356 & 44.4 & 1.336 & 44.0 & 1.397 & 45.2 & 1.390 & 46.0 \\
\hline 8 & TAS-92-34 & 1.52 & 46.8 & 1.442 & 46.1 & 1.420 & 45.6 & 1.486 & 46.9 & 1.478 & 47.7 \\
\hline 9 & HIMSOY-1 & 1.43 & 48.4 & 1.314 & 47.0 & 1.294 & 46.5 & 1.353 & 47.8 & 1.346 & 48.6 \\
\hline 10 & CAT-2722 & 1.54 & 46.8 & 1.412 & 45.4 & 1.390 & 44.9 & 1.454 & 46.2 & 1.447 & 47.0 \\
\hline 15 & KDS-869 & 1.78 & 52.8 & 1.635 & 51.3 & 1.610 & 50.7 & 1.684 & 52.1 & 1.675 & 53.1 \\
\hline 16 & NRC-127 & 1.80 & 50.6 & 1.655 & 49.1 & 1.630 & 48.6 & 1.704 & 50.0 & 1.696 & 50.9 \\
\hline 17 & AGS-432 & 1.66 & 49.5 & 1.527 & 48.0 & 1.503 & 47.6 & 1.572 & 48.9 & 1.564 & 49.7 \\
\hline 18 & CAT-49 & 1.82 & 53.9 & 1.678 & 52.3 & 1.652 & 51.8 & 1.728 & 53.2 & 1.719 & 54.2 \\
\hline 19 & CAT-49586 & 1.75 & 50.6 & 1.607 & 49.1 & 1.582 & 48.6 & 1.655 & 50.0 & 1.646 & 50.9 \\
\hline 20 & JS-20-42 & 1.69 & 52.8 & 1.554 & 51.3 & 1.529 & 50.7 & 1.599 & 52.1 & 1.591 & 53.1 \\
\hline \multirow{3}{*}{\multicolumn{2}{|c|}{$\begin{array}{c}\text { S. Em } \pm \\
\text { CD }(P=0.01) \\
\text { CV }(\%)\end{array}$}} & 0.22 & 1.08 & 0.32 & 0.13 & 0.22 & 0.18 & 0.62 & 0.21 & 0.22 & 0.11 \\
\hline & & 0.63 & 4.35 & 0.98 & 0.34 & 0.68 & 0.62 & 1.93 & 0.63 & 0.69 & 0.36 \\
\hline & & 1.44 & 2.16 & 3.76 & 1.11 & 2.15 & 1.45 & 2.34 & 2.27 & 2.32 & 4.58 \\
\hline
\end{tabular}

Where, EC: electrical conductivity, MII: membrane injury index 
The positive effect of seed amelioration on seedling vigour index was might be due to reserve mobilization of food material, repair and re-synthesis of various enzymes and rapid growth of embryo results in enhanced germination, seedling growth and seedling dry weight in turn increases the seedling vigour index (Khan, 1992).

Besides physiological parameters, significant changes were also observed in biochemical parameters due to genotypes and their seed treatments. Among various treatments $\alpha$ tocopherol recorded the least amount of electrical leachates $(1.199 \mathrm{dS} / \mathrm{cm})$ and membrane injury index $(33.8 \%)$ followed by ascorbic acid (1.217 dS/cm) (34.1\%) and PEG (1.247 dS/cm) (36.8 \%) compared to control (1.283 dS/cm) (38.7 \%) in PB-5. Higher electrical conductivity was reported for potassium iodide treatment in CAT-49 $(1.728 \mathrm{dS} / \mathrm{cm})$ and NRC-127 (1.704 dS/cm) compared to other treatments and genotypes (Table 4).

Irrespective of genotype, antioxidant treatment significantly reduced the electrolyte leachate concentration in all treated seeds of aged genotypes compared to untreated aged seeds. Braccini Adle et al., (2000) in soybean and Khan et al., (1977) have reported that amelioration activates antioxidant enzymes which reduces peroxidation of lipids in seed by stabilizing reactive oxygen species generated in aged seeds thereby results in low damage to membrane and low solute leakage from seed cells due to which increase in electrical conductivity comes under control. These reduced membrane injury index through $\alpha$-tocopherol treatment indicates the repair and re-synthesizing ability of antioxidants and their role in stabilizing seed membrane damage.

Enhanced electrolyte leakage is often indicated as a symptom of stress related membrane injury, caused by the accumulation of reactive oxygen species. Seed amelioration using antioxidants and osmotics found to be significantly alleviated the adverse effect of ROS, which considerably enhanced membrane stability. These results were confirmed by the results of Noreen et al., (2010) in turnip.

The study could be concluded that the antioxidants like $\alpha$-tocopherol $(1 \%)$ and ascorbic acid (1\%) can be recommended as the efficient seed amelioration treatments against the seed deterioration damages in soybean germplasm accessions.

\section{References}

Abdul-Baki, A. A. and Anderson, J.D., 1973, Vigour determination in soybean seed by multiple criteria. Crop Sci., 13: 630633.

Anonymous, 2010, International rules for seed testing. pp 27-42.

Bailly, C., Benamar, A., Corbineau, F. and Come, D., 1998, Free radical scavenging as affected by accelerated ageing and subsequent priming in sunflower seeds. Physiol. Plant, 104: 646-652.

Blum, A. and Ebercon, A., 1981, Cell membrane stability as a measure of drought and heat tolerance in wheat. Crop Sci., 21(1):43-47.

Braccini Adle, Reis, M. S., Moreira, M. A., Sediyama, C. S. and Scapim, C.A. 2000, Biochemical changes associated to soybean seeds osmo-conditioning during storage. Pesqui. Agropecu. Bras., 35(2): 433-447.

Kapoor, N., Arya, A., Siddiqui, A. M., Kumar, H. and Amirn, A., 2011. Physiological and biochemical changes during seed deterioration in aged seeds of rice (Oryza sativa L.). American $J$. Plant Physiol., 6(1): 28-35. 
Kaya, M. D., Okcu, G. and Atak, M., 2006, Seed treatments to overcome salt and drought stress during germination in sunflower (Helianthus annus L.). Eur. J. Agron., 24: 291-295.

Khan, A. A., 1992, Pre-plant physiological conditioning. Horti. Rev., 13: 131-181.

Khan, A. A., Tao, K., Knypl, J. S., Borkowska, B. and Powell, L. E., 1977, Osmotic conditioning of seeds: physiological and biochemical changes. Symposium on Seed Problems in Hortic., 83: 267-278.

Noreen, Z., Ashraf, M. and Akram, N., 2010, Salt induced regulation of some key antioxidant

enzymes

and physio- biochemical phenomena in five diverse cultivars of turnip (Brassica rapa L.). J. Agronomy and Crop Sci., 196(4): 273-285.

Sundaraj, N., Nagaraju, S., Venkataramulu, M.N. and Jaganath, M. K., 1972, Design and analysis of field experiments. UAS, Bangalore, pp 5459.

Taylor, A. G., Allen, P. S., Bennett, M. A., Bradford, K. J., and Misra, M.K., 1998, Seed enhancements. Seed Sci. Res., 8: 245-256.

\section{How to cite this article:}

Shruthi, K., R. Siddaraju, P.J. Devaraju, N. Nethra, Jayarame Gowda and Nanja Reddy, Y.A. 2018. Influence of Various Seed Amelioration Techniques on Physio-Biochemical Changes during Seed Deterioration in Aged Seeds of Soybean [Glycine max (L.) Merill] Mini Core Set. Int.J.Curr.Microbiol.App.Sci. 7(09): 406-413. doi: https://doi.org/10.20546/ijcmas.2018.709.050 